\title{
A forma como o balanço patrimonial é apresentado altera a percepção do usuário externo quanto à interpretação do conteúdo informacional? Uma investigação no contexto brasileiro e cabo-verdiano
}

Does the way that the balance sheet is presented alter the perception of the external user in regard to understanding of informational content? An investigation in the Brazilian and Cape-Verdean contexts.

¿La forma en que se presenta el balance de situación altera la percepción del usuario externo en relación a la interpretación del contenido informacional? Una investigación realizada en Brasil y en Cabo Verde

Valdemir Galvão de Carvalho

Doutorando no UNB / UFPB / UFRN

Professor do Departamento de Administração da UFRN

Endereço: Avenida Cap Mor Gouveia s/n, Bairro Lagoa Nova

CEP: 59.000000 - Natal/RN - Brasil

E-mail: professorvaldemir@hotmail.com

Telefone: (84) 3215-8420

José Jailson da Silva

Doutorando no UNB / UFPB / UFRN

Professor do Departamento de Ciências Contábeis da UFRN

Endereço: Avenida Cap Mor Gouveia s/n, Bairro Lagoa Nova

CEP: 59.000000 - Natal/RN - Brasil

E-mail: professorjailsonufrn@yahoo.com.br

Telefone: (84) 3215-8406

Carlos Jorge Fontainhas Mendes

Doutorando no UNB / UFPB / UFRN

Professor do Departamento de Ciências Contábeis da Universidade Federal de Cabo Verde

Endereço: Avenida Cap Mor Gouveia s/n, Bairro Lagoa Nova

CEP: 59.000000 - Natal/RN - Brasil

E-mail: fontainhas.carlos@gmail.com

Telefone: (84) 3215-8406

\section{José Dionísio Gomes da Silva}

Professor do Doutorado no Doutorando no UNB / UFPB / UFRN

Endereço: Avenida Cap Mor Gouveia s/n, Bairro Lagoa Nova

CEP: 59.000000 - Natal/RN - Brasil

E-mail: dionisio@ufrnet.br

Telefone: (84) 3215-8406

Artigo recebido em 10/05/2013. Revisado por pares em 07/09/2013. Reformulado em 08/10/2013. Recomendado para publicação em 09/11/2013 por Sandra Rolim Ensslin (Editora Científica). Publicado em 15/12/2013. 


\title{
Resumo
}

Esta pesquisa analisa se a forma como o Balanço Patrimonial apresentado altera a percepção dos acadêmicos brasileiros e cabo-verdianos quanto à interpretação do conteúdo informacional para tomada da decisão. Foi realizado um experimento com modelos distintos de balanço patrimonial, um adotado no padrão brasileiro e outro europeu. Foram aplicados 152 questionários em acadêmicos da UFRN e 64 da UNICV. Os resultados da estatística descritiva e análise da variância sugerem presença do efeito propriedade e que a subordinação à forma pode influenciar na compreensão do conteúdo informacional, e a forma de apresentação das demonstrações pode afetar a interpretação do usuário.

Palavras-chave: Contabilidade Comportamental. Demonstrações contábeis. Efeito Framing. Comportamento do usuário externo.

\begin{abstract}
This study seeks to investigate whether the way the balance sheet is presented alters the perception of Brazilian and Cape-Verdean academicians when it comes to the understanding of informational content for decision making. An experiment was carried out with different balance sheet formats: one which was adapted according to the Brazilian format, and the other was European. 152 questionnaires were administered to academicians from Universidade Federal do Rio Grande do Norte and other 64 were administered to academicians from Universidade do Cabo Verde. Descriptives and analysis of variance suggest the occurrence of framing effect. In addition, the statistical findings show that the subordination to the format can influence understanding on informational content, and the way that the statements are presented can influence the user's interpretation.
\end{abstract}

Keywords: Behavioral accounting. Accounting statements. Framing effect. Behavior of the external user.

\section{Resumen}

Esta investigación analiza si la forma en que el balance presentado altera la percepción de los académicos brasileños y caboverdianos en la interpretación del contenido informacional para la tomada de decisión. Hemos llevado a cabo un experimento con distintos modelos de balance de situación, uno con el patrón brasileño y otro con la norma europea. 152 cuestionarios fueron aplicados en académicos de UFRN y 64 en académicos de UNICV. Los resultados de la estadística descriptiva y el análisis de la varianza sugieren la presencia del efecto de dotación y que la subordinación a la forma puede influenciar en la comprensión del contenido informacional y que la presentación de los informes contables podría afectar la interpretación del usuario.

Palabras clave: Contabilidad comportamental. Informes contables. Teoría del encuadre. Comportamiento del usuario externo. 


\section{Introdução}

A formação do ser humano inclui a busca em cobrir as necessidades individuais e coletivas, como a sobrevivência, o saber, a segurança, o bem-estar, o domínio da natureza e outros. Essas necessidades o impulsionam, enquanto ser pensante, para a racionalização dos meios e das formas de atingir seus objetivos, enfrentando os desafios que modificam o conhecimento e a trajetória de toda vida na terra.

Essa formação é construída com a busca de alcançar os objetivos e suplantar os desafios que entravam o desenvolvimento humano. Os aspectos psicológicos e sociológicos contribuem para a modelagem dos relacionamentos e dos comportamentos na sociedade. Entre os desafios podem ser citados o de aprender a contar e o de aprender a utilizar a informação. Neste ínterim, a contabilidade pode ser utilizada como ferramenta, tentando descrever (teoria descritiva) e explicar o comportamento das grandezas econômicas no âmbito da informação produzida pelas práticas contábeis, prestando relevante contribuição para o estudo dos aspectos qualitativos da informação manuseada pelos operadores da contabilidade.

Práticas podem ser compreendidas como orientações gerais de regra de conduta. Assim como o sentido de normas e procedimentos é derivado das práticas, aplicado de acordo com as circunstâncias para seus usuários, os quais se relacionam com o sistema contábil processando registros e introduzindo ou modificando práticas e procedimentos.

Essa área de estudo, envolvendo viés e tendência de operadores e usuários, vem sendo denominada Contabilidade Comportamental e seu objeto é constituído pelo relacionamento do comportamento humano com a informação produzida pela contabilidade.

As alterações nos métodos de depreciação ou técnicas de inventário conduzem a diferentes valores de lucro, demonstrando um comportamento que influencia o desempenho da firma, em que é possível verificar que operadores da contabilidade e usuários da informação influenciam procedimentos contábeis direcionados produzindo benefícios e recompensas (BELKAOUI, 1989).

A Contabilidade Comportamental é uma interface da contabilidade com a ciência social, preocupando-se com a maneira como o comportamento humano influencia dados contábeis e decisões de negócios e como a informação contábil afeta decisões de negócio e o comportamento humano. A ciência comportamental se preocupa com a explicação e predição do comportamento humano, enquanto que a Contabilidade Comportamental se preocupa com o relacionamento entre o comportamento humano e as práticas contábeis (SIEGEL; RAMANAUSKAS, 1989).

Assim, o enfoque comportamental em relação à teoria da contabilidade tem estimulado buscas, por meio de estudantes e de profissionais do mercado, de objetivos fundamentais para a contabilidade, bem como entender a reação de investidores, aportadores e gestores, diante de procedimentos e informações diferentes (HENDRIKSEN; BREDA, 1999).

$\mathrm{O}$ relacionamento dos operadores da contabilidade com as entidades e dos procedimentos com as práticas é intenso, podendo originar vieses que beneficiem ou prejudiquem pessoas ou instituições com interesse no desempenho dessas entidades. Referidos procedimentos têm impacto no desempenho da entidade, afetando de forma direta no patrimônio da contabilidade, bem como o valor dos compromissos sociais como tributos que são de interesse da sociedade, lucros que são a remuneração do capital, e benefícios e recompensas que são remunerações dos gestores e empregados, como interesses individuais ou coletivos. 
Do lado dos usuários, os dados contidos nas demonstrações contábeis podem alimentar o desejo de participar do negócio como investidor, aportador ou colaborador ou ainda de forma contrária, provocar a decisão de sair do negócio. Essas percepções do usuário podem influenciar procedimentos contábeis posteriores ou podem ser influenciadas por procedimentos contábeis anteriores. Esse contexto de percepções também está dentro do objeto de estudo da Contabilidade Comportamental.

Diante do acima exposto indaga-se: a forma como o Balanço Patrimonial é apresentado altera a percepção dos acadêmicos brasileiros e cabo-verdianos quanto à interpretação do conteúdo informacional para a tomada da decisão?

Esta pesquisa tem por objetivo analisar se a forma como o Balanço Patrimonial é apresentado altera a percepção dos acadêmicos brasileiros e cabo-verdianos quanto à interpretação do conteúdo informacional para a tomada da decisão.

\section{Referencial Teórico}

\subsection{A Contabilidade Comportamental e o Processo Decisório}

Diversos são os aspectos que permeiam o processo decisório e que devem ser considerados na busca de sua compreensão. Tal entendimento é mais comumente explorado em pesquisas no campo da administração e da economia, valendo-se, inclusive, dos aspectos abordados em psicologia para poder compreender o comportamento do tomador de decisão (SIMON, 1965; SIMON, 1979). Mais recentemente, tais aspectos estão sendo explorados no campo das finanças comportamentais (KIMURA, 2003, p. 3).

Já no campo da contabilidade financeira, a preocupação principal que norteou as pesquisas nos últimos anos está centrada nos aspectos do reconhecimento, mensuração e evidenciação das informações por ela geradas, com o objetivo de subsidiar o processo decisório, sem, no entanto, buscar explorar outras variáveis desse processo, como por exemplo, o uso de tais informações.

Simon (1965) afirma que o homem é um ser com racionalidade limitada. Aspectos como o comportamento do usuário perante essas informações e itens relevantes que envolvem o processo decisório, entre outros, têm sido pouco explorados pelos pesquisadores na área contábil, sendo muitas vezes objeto de críticas, inclusive aquelas de natureza financeira (LIBBY; BLOOMFIELD; NELSON, 2002, p. 781).

A Contabilidade Comportamental está também interessada em saber como os efeitos cognitivos podem ser alterados por mudanças na maneira pela qual a contabilidade é realizada e como os relatórios contábeis e os procedimentos podem ser usados mais eficazmente para ajudar indivíduos e organizações a atingir os seus objetivos. Ariely (2008), Tversky e Kahneman (1981), Baker e Nofsinger (2002), Barber e Odean (1999), Bazerman (2004), entre outros, denominaram esses efeitos de vieses e/ou efeitos comportamentais.

Embora a Ciência Comportamental seja um subconjunto das ciências sociais, a Contabilidade Comportamental é um subconjunto de ambas que considera a relevância dos efeitos cognitivos, na tomada de decisão. Assim sendo, Siegel e Ramanauskas-Marconi (1989) consideram que o campo de aplicação da Contabilidade Comportamental pode ser dividido em três áreas gerais: a primeira relacionada ao efeito do comportamento humano, sobre a concepção, construção e utilização do sistema de contabilidade; a segunda se refere ao 
efeito do sistema de contabilidade sobre o comportamento humano; e a terceira mais ligada diretamente ao estudo, são os métodos de previsão e as estratégias para alterar o comportamento humano.

Essa área da Contabilidade Comportamental está preocupada com a forma como o sistema de contabilidade pode ser utilizado para influenciar comportamentos. Desse modo, o processo decisório ganha um caráter comportamental. Nesse sentido, diante da complexidade e da incerteza, tomadores de decisão normalmente utilizam heurísticas, fórmulas práticas que reduzem o tempo e a complexidade da decisão, que por estarem sujeitas a uma série de vieses, comprometem em certa medida a sua racionalidade, entendida como o processo de tomada de decisões que conduziria ao resultado ótimo: o que apresenta a maior utilidade esperada (GERLETTI; SAUAIA, 2008).

\subsection{Efeito Framing e a Cognição Numérica}

O efeito framing faz parte da Prospect Theory, desenvolvido por Kahneman e Tversky (1979) como sendo uma abordagem das Finanças Comportamentais alternativa à Teoria da Utilidade. A Prospect Theory defende que o processo de escolha pode ser dividido em duas fases: revisão, que consiste na análise das opções existentes, e avaliação, em que são avaliadas as opções antes analisadas. O efeito framing se verifica na fase de revisão em que as opções não analisadas. Kahneman e Tversky (1979) argumentam que a forma como as informações são apresentadas influenciam fortemente no momento da tomada de decisão.

Sendo as demonstrações contábeis compostas predominantemente por números, a forma como esses números são apresentados se relaciona com o processo de cognição numérica. Os processos psicológicos subjacentes à utilização dos conceitos de número começaram a ser investigados por psicólogos nas décadas de 1960 e 1970.

Um dos primeiros estudos de comparação de números foi realizado por Moyer e Landauer em 1967, em que mediram a velocidade de decisão dos estudantes universitários para selecionar qual o número maior quando apresentados a dois números simples, de apenas um dígito (HINRICHS; YURKO; HU, 1981).

O foco dos estudos em cognição numérica são questões de representação, ou seja, de que forma os números são representados no sistema cognitivo e qual o papel dos vários formatos de representação no processamento numérico (McCLOSKEY; MACARUSO, 1995, p. 357; VANHUELE; DRÈZE, 2002).

Existe uma distinção entre os componentes do processamento dos números arábicos e os componentes do processamento numérico verbal nos subsistemas de compreensão e produção numérica (McCLOSKEY; CARAMAZZA; BASILI, 1985).

Segundo McCloskey e Macaruso (1995, p. 353), "as principais formas de representação numérica são: representação semântica; representação verbal (fonológica e grafêmica); e a representação arábica”. Nesse contexto, McCloskey (1992) aborda a questão de se a representação semântica dos números é precisa ou aproximada, enquanto Dehaene (1992) questiona sobre se os números são representados como unidades inteiras no nível semântico ou são representados decompostos em partes. 
No que se refere à comparação numérica, a codificação dos números pode ser dividida em analógica ou digital; a representação analógica é contínua, enquanto que a representação digital é discreta (DEHAENE; DUPOUX; MEHLER, 1990, p. 17). O modelo analógico ou holístico sugere que quando dois números com multidígitos são comparados, o processo de codificação quantifica esses números como um todo, usando uma representação interna de grandeza, chamada linha numérica. Por outro lado, no modelo digital, nenhum cálculo de grandeza é necessário, pois os dígitos são comparados como símbolos. Ou seja, o processo de codificação se dá dígito por dígito.

Ao ser realizada uma comparação entre dois números, Dehaene, Dupoux e Mehler (1990) explicam que, no modelo digital, os indivíduos primeiro extraem apenas as dezenas dos dois números e então os comparam; posteriormente, eles recorrem à comparação entre as unidades somente se as duas dezenas forem iguais. Alternativamente, de acordo com o modelo analógico, a comparação não acontece no nível dos dígitos. Ao invés disso, o input do símbolo seria transformado em uma representação de sua grandeza e só então a comparação seria feita. Desse modo, a principal diferença entre os modelos se dá nos estágios de processamento em que a comparação dos números acontece.

Importantes estudos, tanto nacional como internacional, já foram realizados abordando questões relacionadas à Contabilidade Comportamental como, por exemplo, os estudos de Rutledge (1995) que explorou os potenciais efeitos moderadores da ocorrência do efeito framing em informações oriundas da Contabilidade Gerencial para decisões relevantes, destacando o efeito recente das informações nas tomadas de decisões; Springer e Borthick (2007), os quais conduziram um trabalho que apontou que os estudantes de Contabilidade se sentem atraídos por tarefas que envolvem conflitos cognitivos; Cardoso e Riccio (2005), que testaram o efeito da variável experiência profissional na minimização da ocorrência do framing; Cardoso e Riccio (2005), Cardoso et al. (2007), Silva e Lima (2007), que conduziram estudos com o objetivo de traçar relações entre a maneira como são tratadas e apresentadas as informações contábeis e o efeito framing; Nascimento, Ribeiro e Junqueira (2008) destacam a ligação entre a Contabilidade Gerencial e o comportamento humano, com ênfase nas abordagens da área de psicologia, que é equivalente à abordagem comportamental dada à Contabilidade por Hendriksen e Van Breda (1999).

\section{Metodologia}

O método é o caminho seguido para se atingir os objetivos propostos. Segundo Matias-Pereira (2010), "trata-se do conjunto de procedimentos utilizados de forma regular, passível de ser repetido, para alcançar objetivo material ou conceitual e compreender o processo de investigação" (MATIAS-PEREIRA, 2010, p. 27).

\subsection{Classificação da Pesquisa}


Quanto a sua natureza, a presente pesquisa pode ser classificada de pesquisa básica, visto que objetiva gerar conhecimentos novos e úteis para o avanço da ciência, sem aplicação prática prevista (SILVA; MENEZES, 2005).

Quanto à forma de abordagem do problema, a pesquisa é quantitativa, uma vez que a análise requer o uso de recursos e de técnicas estatísticas tais como: média, desvio-padrão, coeficiente de correlação, análise de regressão (SILVA; MENEZES, 2005).

Quanto aos fins, a investigação é explicativa, visto que tem como principal objetivo estabelecer a relação entre a forma que o relatório contábil é apresentado e a sua interpretação. A pesquisa explicativa visa identificar os fatores que determinam ou contribuem para ocorrência dos fenômenos. Assim, aprofunda o conhecimento da realidade e explica a razão, o "porquê" das coisas (GIL, 2002, p. 43).

Quanto aos meios de investigação ou procedimentos técnicos, trata-se de uma pesquisa experimental que se caracteriza por manipular diretamente as variáveis relacionadas com o objeto de estudo e, desse modo, sugerir de que maneira ou por que causa o fenômeno é produzido (GIL, 2002).

\subsection{Universo e Amostra}

Esta pesquisa tem por objetivo analisar se a forma como o Balanço Patrimonial é apresentado altera a percepção dos acadêmicos brasileiros e cabo-verdianos quanto à interpretação do conteúdo informacional para a tomada de decisão. Para isso, foi realizado um experimento com dois modelos distintos do Balanço Patrimonial, o modelo adotado no Brasil, proposto pela Comissão de Valores Mobiliários - CVM e o modelo adotado em alguns países da Europa e da África, adotando-se Cabo Verde para a coleta da amostra.

Para efeito desta pesquisa, o universo compreende alunos de Contabilidade de turmas concluintes que já haviam cursado as disciplinas de administração financeira e análise das demonstrações financeiras pertencentes a duas turmas do curso de graduação em Contabilidade da Universidade Federal do Rio Grande do Norte - UFRN, onde foram aplicados 152 questionários sendo 76 em duas turmas da UFRN, com o balanço patrimonial no modelo europeu e $76 \mathrm{em}$ duas turmas da UFRN, com o balanço patrimonial no modelo brasileiro com o padrão exigido pela CVM. A mesma metodologia foi adotada para a coleta de dados em Cabo Verde com duas turmas do curso de graduação em Contabilidade da Universidade Pública de Cabo Verde - UNICV, onde foram coletados 64 questionários.

A aplicação do instrumento de pesquisa foi realizada no período de maio a junho de 2012, de forma presencial nas turmas de graduação em Contabilidade que estavam cursando ou já haviam cursado as disciplinas de administração financeira e análise das demonstrações financeiras no curso de graduação em Contabilidade da UNICV e da UFRN.

Tabela 1 - Distribuição das amostras

\begin{tabular}{l|c|c}
\hline \multicolumn{1}{c|}{ Demonstrações financeiras } & Grupos & Turmas \\
\hline Modelo padrão CVM & $1^{\mathrm{a}}$ amostra & 76 alunos da UFRN \\
\hline Modelo padrão europeu & $2^{\mathrm{a}}$ amostra & 76 alunos da UFRN \\
\hline Modelo padrão CVM & $3^{\mathrm{a}}$ amostra & 32 alunos da UNICV \\
\hline Modelo padrão europeu & $4^{\mathrm{a}}$ amostra & 32 alunos da UNICV \\
\hline
\end{tabular}

Fonte: Elaborada pelos autores. 


\subsection{Técnica de Coleta de Dados}

Para o presente estudo, foi elaborado como instrumento de pesquisa questionário estruturado, que possui perguntas referentes ao perfil dos participantes em relação à idade, ao gênero e à renda familiar de acordo com a distribuição da classe econômica, e perguntas referentes ao comportamento do usuário das informações contábeis na interpretação da comparação das demonstrações contábeis quanto a sua forma de apresentação, quanto à distribuição e disposição das contas no Balanço Patrimonial em relação ao seu grau de liquidez, visto que no Brasil as contas são distribuídas na ordem do grau de liquidez, enquanto que em alguns países europeus e africanos, no Balanço Patrimonial, as contas são distribuídas na ordem inversa do seu grau de liquidez.

O instrumento de pesquisa foi elaborado com escala de representação gráfica, em que o respondente verifica sua resposta em qualquer ponto de uma linha contínua em escala milimétrica, possibilitando uma maior aleatoriedade nos dados favorecendo o tratamento estatístico. Segundo Cooper e Schindler (2003, p. 201), a escala de representação gráfica permite aos pesquisadores discernirem diferenças sutis.

A pesquisa também utilizou dados secundários, informações referentes às demonstrações financeiras de uma empresa distribuidora de combustíveis do Brasil e outra de Cabo Verde coletados no site <http://www.cvm.gov.br/>, referente às demonstrações financeiras do ano de 2011.

A pesquisa investigou diversos questionamentos sobre o comportamento do usuário da informação contábil; dentre estes, tratou-se das questões referentes ao efeito propriedade e à teoria da subordinação à forma.

Para verificar evidências da existência desses efeitos, investigamos o comportamento dos usuários das informações contábeis, conforme demonstrado no quadro abaixo:

\section{Quadro 1- Questões Investigadas}

\begin{tabular}{|c|c|c|}
\hline Efeito comportamental & Investigação & $\begin{array}{c}\text { Sinal } \\
\text { esperado }\end{array}$ \\
\hline Efeito propriedade & $\begin{array}{l}\text { O usuário prefere outro tipo de representação gráfica do relatório } \\
\text { financeiro. }\end{array}$ & $(-)$ \\
\hline \multirow{3}{*}{$\begin{array}{l}\text { Subordinação } \\
\text { à forma }\end{array}$} & $\begin{array}{l}\text { A forma como as demonstrações financeiras são apresentadas } \\
\text { influenciam no julgamento dos usuários das informações contábeis na } \\
\text { tomada da decisão. }\end{array}$ & $(+)$ \\
\hline & $\begin{array}{l}\text { A forma como as demonstrações financeiras são apresentadas } \\
\text { influenciam na compreensão do conteúdo informacional pelos } \\
\text { usuários das informações contábeis. }\end{array}$ & $(+)$ \\
\hline & $\begin{array}{l}\text { A forma como as demonstrações financeiras são apresentadas } \\
\text { influenciam na análise das demonstrações financeiras por meio de } \\
\text { índices financeiros. }\end{array}$ & $(-)$ \\
\hline
\end{tabular}

Fonte: Elaborado pelos autores.

\subsection{Análise e Tratamento dos Dados}

Para a tabulação, o tratamento e a análise dos dados, utilizou-se o software Gretl, por meio da análise da estatística descritiva, análise de correlação, análise do teste student e a 
análise de variância (ANOVA). Essas análises possibilitaram verificar se existe diferença entre as médias das variáveis para as hipóteses da pesquisa.

\section{Análise dos Resultados}

\subsection{Perfil Sócio-Demográfico}

De acordo com a Tabela 2, verifica-se que nesta pesquisa a maioria dos alunos em contabilidade possui a idade entre 21 e 30 anos e é do gênero masculino.

Tabela 2 - Perfil sócio-demográfico das amostras

\begin{tabular}{c|c|c|c|c|c|c}
\hline \multirow{4}{*}{ Idade } & Grupos & $\mathbf{2 0}$ & $\mathbf{2 1}$ a 25 & $\mathbf{2 6}$ a 30 & $\mathbf{3 1}$ a 39 & 40 a 49 \\
\cline { 2 - 7 } & $1^{\mathrm{a}}$ amostra & $17,15 \%$ & $52,58 \%$ & $19,21 \%$ & $5,03 \%$ & $3,03 \%$ \\
\cline { 2 - 7 } & $2^{\mathrm{a}}$ amostra & $7,06 \%$ & $56,58 \%$ & $23,24 \%$ & $13,12 \%$ & $0,00 \%$ \\
\cline { 2 - 7 } & $3^{\mathrm{a}}$ amostra & $2,45 \%$ & $72,57 \%$ & $7,43 \%$ & $14,28 \%$ & $3,27 \%$ \\
\cline { 2 - 7 } & $4^{\mathrm{a}}$ amostra & $3,42 \%$ & $61,58 \%$ & $15,00 \%$ & $17,00 \%$ & $3,00 \%$ \\
\hline \multirow{5}{*}{ Gênero } & Grupos & Masculino & Feminino & - & - & - \\
\cline { 2 - 7 } & $1^{\mathrm{a}}$ amostra & $52,55 \%$ & $47,45 \%$ & - & - & - \\
\cline { 2 - 7 } & $2^{\mathrm{a}}$ amostra & $58,64 \%$ & $41,36 \%$ & - & - & - \\
\cline { 2 - 7 } & $3^{\mathrm{a}}$ amostra & $50,00 \%$ & $50,00 \%$ & - & - & - \\
\cline { 2 - 7 } & $4^{\mathrm{a}}$ amostra & $46,00 \%$ & $54,00 \%$ & - & - & - \\
\hline
\end{tabular}

Fonte: Dados da pesquisa.

\subsection{Análise de Correlação}

A Tabela 3 apresenta o resultado da matriz de correlação de Pearson para a hipótese nula de não correlação entre as variáveis das duas amostras aplicadas no Brasil. O resultado sugere que não há correlação entre as duas amostras, exceto quanto à forma de objetividade da apresentação do relatório financeiro, com baixa correlação negativa de $-0,36085$ com $p$ value $\geq 0,01955$ e o ROE que também apresentou um baixo índice de correlação negativa 0,3666 , com $p$-value $\geq 0,0179$.

Tabela 3 - Correlação de Pearson entre as variáveis das duas amostras no padrão CVM

\begin{tabular}{l|c|c|c}
\multicolumn{1}{c|}{ Questões da Pesquisa } & Correlação & Teste t & $\begin{array}{c}\text { p-value } \\
\text { bilateral }\end{array}$ \\
\hline O relatório é apresentado de forma clara & $-0,1044$ & $-0,5836$ & 0,5637 \\
\hline O relatório é apresentado de forma objetiva & $\mathbf{- 0 , 3 6 0 8}$ & $-2,1543$ & $\mathbf{0 , 0 3 9 1}$ \\
\hline O relatório é apresentado de forma adequada & $-0,1629$ & $-0,9047$ & 0,3728 \\
\hline O usuário prefere outro tipo de apresentação gráfica do relatório & 0,04507 & 0,2471 & 0,8065 \\
\hline $\begin{array}{l}\text { A forma como as demonstrações financeiras são apresentadas } \\
\text { influenciam no julgamento }\end{array}$ & 0,2083 & 1,1860 & 0,2446 \\
\hline $\begin{array}{l}\text { A forma como as demonstrações financeiras são apresentadas } \\
\text { influenciam na compreensão }\end{array}$ & $-0,0565$ & $-0,3149$ & 0,7549 \\
\hline Efeito propriedade & 0,2099 & 1,1763 & 0,2487 \\
\hline Consigo identificar a situação de liquidez corrente & 0,0143 & 0,0799 & 0,9368 \\
\hline É possível identificar a situação do ROA & $-0,1087$ & $-0,5886$ & 0,5607 \\
\hline É possível identificar a situação de ROE & $\mathbf{- 0 , 3 6 6 6}$ & $-2,1941$ & $\mathbf{0 , 0 3 5 8}$ \\
\hline
\end{tabular}




\begin{tabular}{l|l|l|c}
\hline Consigo identificar a estrutura de capital & $-0,0703$ & $-0,3926$ & 0,6973 \\
\hline Fonte: Dados da pesquisa.
\end{tabular}

\subsection{Estatística Descritiva e Análise do Teste T (Student)}

Os dados da Tabela 4 mostram o resultado da estatística descritiva e do teste student para a hipótese nula de que a diferença de médias é igual a zero. Para as amostras brasileiras, o resultado sugere que há diferenças entre as médias da percepção dos alunos da UFRN em relação à clareza dos relatórios financeiros padrão CVM e padrão europeu, $p$-value $\geq 0,0138 \mathrm{e}$ o mesmo foi observado para as amostras dos alunos cabo-verdianos da UNICV, $p$-value $\geq$ 0,0091 .

Tabela 4 - Clareza do relatório

\begin{tabular}{c|c|c|c|c|c|c}
\hline Grupos amostrais & Média & $\boldsymbol{\Sigma}$ & $\begin{array}{c}\text { Erro } \\
\text { padrão }\end{array}$ & $\begin{array}{c}\text { Intervalo de } \\
\text { confiança }\end{array}$ & \multirow{2}{*}{ Teste t } & p-value \\
\hline $1^{\mathrm{a}}$ amostra $(\mathrm{n}=76)$ & 6,9141 & 3,9689 & 0,6909 & 5,5068 a 8,3214 & 2,2534 & 0,0138 \\
\hline $2^{\mathrm{a}}$ amostra $(\mathrm{n}=76)$ & 4,7505 & 3,8302 & 0,6668 & 3,3924 a 6,1086 & & \\
\hline $3^{\mathrm{a}}$ amostra $(\mathrm{n}=32)$ & 3,3129 & 2,3950 & 0,5495 & 2,1579 a 4,4666 & 2,4763 & 0,0091 \\
\hline $4^{\mathrm{a}}$ amostra $(\mathrm{n}=32)$ & 5,5157 & 3,0509 & 0,6999 & 4,0453 a 6,9863 & & \\
\hline
\end{tabular}

Fonte: Dados da pesquisa.

A Tabela 5 mostra o resultado da estatística descritiva e do teste student em relação à percepção da forma objetiva de apresentação dos relatórios financeiros no padrão CVM e no padrão europeu. Os resultados das amostras dos alunos da UFRN e da UNICV foram semelhantes, sugerindo que há diferenças entre as médias, portanto a não aceitação da hipótese nula de que a diferença de médias é igual a zero com $p$-value $\geq 0,000027$ e 0,0416 , respectivamente.

Tabela 5 - Objetividade do relatório

\begin{tabular}{|c|c|c|c|c|c|c|}
\hline Grupos amostrais & Média & $\boldsymbol{\sigma}$ & $\begin{array}{c}\text { Erro } \\
\text { padrão }\end{array}$ & $\begin{array}{l}\text { Intervalo de } \\
\text { confiança }\end{array}$ & Teste $\mathbf{t}$ & p-value \\
\hline $1^{\mathrm{a}}$ amostra $(\mathrm{n}=76)$ & 7,5909 & 3,3027 & 0,5749 & 6,4198 a 8,7621 & \multirow{2}{*}{4,3207} & \multirow{2}{*}{0,00003} \\
\hline $2^{\mathrm{a}}$ amostra $(\mathrm{n}=76)$ & 4,0515 & 3,3520 & 0,5835 & 2,8629 a 5,2401 & & \\
\hline $3^{\mathrm{a}}$ amostra $(\mathrm{n}=32)$ & 3,7632 & 3,2878 & 0,75427 & 2,1785 a 5,3478 & \multirow{2}{*}{1,7822} & \multirow{2}{*}{0,0416} \\
\hline $4^{\mathrm{a}}$ amostra $(\mathrm{n}=32)$ & 5,6053 & 3,0803 & 0,7067 & 4,1206 a 7,0899 & & \\
\hline
\end{tabular}

Fonte: Dados da pesquisa.

Quanto à forma adequada do relatório financeiro, verificou-se que a diferença de médias foi igual a zero, com p-valor $\geq 0,0766$. Esse resultado sugere que, para as amostras dos alunos da UFRN, não houve diferença estatística significante entre as médias da percepção dos alunos quanto à forma de apresentação adequada dos relatórios financeiros no padrão CVM e no padrão europeu. Entretanto, os resultados das amostras dos alunos da UNICV apresentaram diferença estatística significante com $p$-value $\geq 0,0049$, corroborando os estudos envolvendo as relações entre a maneira como são tratadas e apresentadas as informações contábeis e o efeito framing (CARDOSO; RICCIO, 2005; CARDOSO et al., 2007; SILVA; LIMA, 2007).

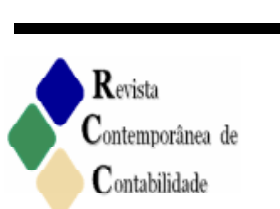


Foi verificado, de acordo com a Tabela 6, se o usuário prefere outro tipo de representação gráfica de relatório financeiro, as diferenças entre as médias foram estatisticamente significantes, com $p$-value $\geq 0,0011$. Isso sugere que, nas amostras brasileiras dos alunos da UFRN, existem diferenças entre as médias e que os mesmos preferem a demonstração no padrão CVM frente à demonstração no padrão europeu, evidenciando a presença do efeito propriedade. Do mesmo modo, as amostras dos alunos cabo-verdianos da UNICV também apresentaram diferença estatística significante com $p$-value $\geq 0,0004$, evidenciando que os mesmos preferem a demonstração financeira padrão europeu frente à demonstração financeira no padrão CVM.

Tabela 6 - Preferência quanto à apresentação do relatório

\begin{tabular}{c|c|c|c|c|c|c}
\hline Grupos amostrais & Média & $\boldsymbol{\sigma}$ & $\begin{array}{c}\text { Erro } \\
\text { padrão }\end{array}$ & $\begin{array}{c}\text { Intervalo de } \\
\text { confiança }\end{array}$ & \multirow{2}{*}{ Teste t } & \multirow{2}{*}{$\boldsymbol{p}$-value } \\
\hline $1^{\mathrm{a}}$ amostra $(\mathrm{n}=76)$ & 5,6222 & 4,4208 & 0,7696 & 4,0547 a 7,1898 & \multirow{2}{*}{$-3,1959$} & \multirow{2}{*}{0,0011} \\
\hline $2^{\mathrm{a}}$ amostra $(\mathrm{n}=76)$ & 8,4844 & 2,5110 & 0,4439 & 7,5790 a 9,3897 & \\
\hline $3^{\mathrm{a}}$ amostra $(\mathrm{n}=32)$ & 3,5693 & 3,3753 & 0,7744 & 1,9425 a 5,1961 & \multirow{2}{*}{2,7243} & \multirow{2}{*}{0,0004} \\
\hline $4^{\mathrm{a}}$ amostra $(\mathrm{n}=32)$ & 6,3947 & 3,0073 & 0,6899 & 4,9453 a 7,8442 & \\
\hline
\end{tabular}

Fonte: Dados da pesquisa.

Quanto à forma como as demonstrações financeiras são apresentadas, influenciam no julgamento dos usuários das informações contábeis na tomada da decisão; o resultado do teste $t$ sugere que para as amostras dos alunos brasileiros não há diferença estatística significante entre as médias, $p$-value $\geq 0,1404$. O resultado da ANOVA não rejeitou a hipótese nula de que as variâncias são iguais $p$-value $\geq 0,2437$. O mesmo se observa também para as amostras dos alunos cabo-verdianos da UNICV que também apresentaram diferença estatística significante com $p$-value $\geq 0,0834$, evidenciando que os mesmos preferem a demonstração financeira padrão europeu frente à demonstração financeira no padrão CVM, demonstrando que o efeito da variável não minimiza a ocorrência do framing (CARDOSO; RICCIO, 2005), o que sugere uma ligação entre a Contabilidade Gerencial e o comportamento humano, no momento da tomada de decisão (NASCIMENTO; RIBEIRO; JUNQUEIRA, 2008).

Em relação à forma como as demonstrações financeiras são apresentadas, influencia na compreensão do conteúdo informacional pelos usuários das informações contábeis. Foi verificado pelo teste $t$ que, para as amostras brasileiras, não há diferença estatística significante entre as médias, $p$-value $\geq 0,3979$. O resultado da ANOVA não rejeitou a hipótese nula de que as variâncias são iguais, $p$-value $\geq 0,1333$. Entretanto, o mesmo não se observa para as amostras dos alunos cabo-verdianos da UNICV, pois os resultados apresentaram diferença estatística significante com $p$-value $\geq 0,0034$ e a ANOVA rejeitou a hipótese nula de que as variâncias são iguais, $p$-value $\geq 0,02336$, sugerindo que a forma como as demonstrações financeiras são apresentadas pode influenciar na compreensão do conteúdo informacional pelos usuários, retratando nos achados de Springer e Borthick (2007) que estudantes de Contabilidade se sentem atraídos por tarefas que envolvem conflitos cognitivos.

Quanto à análise das demonstrações financeiras por meio de índices financeiros, utilizando-se as demonstrações no padrão CVM e no padrão europeu, foi questionado se é possível identificar o ROA e o ROE e se é possível conseguir identificar a liquidez e a estrutura de capital. No caso das amostras da UFRN, verificou-se que em relação ao índice de 
Liquidez Corrente e para o índice de Estrutura de Capital não houve significância estatística entre as médias, com $p$-value $\geq 0,1305$ e $\geq 0,3238$, respectivamente; em relação ao ROA e ROE, os resultados sugerem que há diferença estatística significante com $p$-value $\geq 0,03075 \mathrm{e}$ $p$-value $\geq 0,0231$, respectivamente. Enquanto que na percepção dos alunos cabo-verdianos da UNICV, os resultados sugerem a mesma tendência que em relação ao índice de Liquidez Corrente e para o índice de Estrutura de Capital não houve significância estatística entre as médias, com $p$-value $\geq 0,4338$ e $\geq 0,4514$, respectivamente. Mas, em relação ao ROA e ROE, diferentemente da amostra brasileira, os resultados sugerem que não há diferença estatística significante com $\mathrm{p}$-valor $\geq 0,4869$ e $\mathrm{p}$-valor $\geq 0,4028$, respectivamente.

\subsection{Análise da variância (ANOVA)}

A Tabela 7 mostra o resultado da ANOVA para a hipótese nula de que as variâncias das populações são iguais com p-valor $\geq 0,05$. O resultado indica que na percepção dos alunos brasileiros, a clareza dos relatórios financeiros padrão CVM e padrão europeu são diferentes, o que sugere a não rejeição da hipótese nula de que as variâncias são iguais para as duas amostras. O mesmo também se verifica na amostra com os alunos cabo-verdianos.

Tabela 7 - Clareza do relatório

\begin{tabular}{c|c|c|c}
\hline Amostras da UFRN & Variância & Estatística $\boldsymbol{F}$ & p-value \\
\hline $1^{\mathrm{a}}$ amostra $(\mathrm{n}=76)$ & 15,7519 & \multirow{2}{*}{1,0737} & 0,4209 \\
\hline $2^{\mathrm{a}}$ amostra $(\mathrm{n}=76)$ & 14,6705 & & \\
\cline { 1 - 2 } Amostras da UNICV & Variância & Estatística $\boldsymbol{F}$ & $\boldsymbol{p}$-value \\
\hline $3^{\mathrm{a}}$ amostra $(\mathrm{n}=32)$ & 5,362 & \multirow{2}{*}{1,6227} & 0,1567 \\
\hline $4^{\mathrm{a}}$ amostra $(\mathrm{n}=32)$ & 9,3081 & &
\end{tabular}

Fonte: Dados da pesquisa.

A Tabela 8 mostra o resultado da ANOVA para a hipótese nula de que as variâncias das populações são iguais ao valor $\geq 0,05$. O resultado sugere que na percepção dos alunos brasileiros, os relatórios financeiros no padrão CVM e no padrão europeu são diferentes quanto à forma objetiva de apresentação. Isso indica a não rejeição da hipótese nula de que as variâncias são iguais para as duas amostras. E o mesmo também se observa para a amostra com os alunos cabo-verdianos.

Tabela 8 - Objetividade do relatório

\begin{tabular}{c|c|c|c}
\hline Amostras da UFRN & Variância & Estatística $\boldsymbol{F}$ & p-value \\
\hline $1^{\mathrm{a}}$ amostra $(\mathrm{n}=76)$ & 10,9081 & \multirow{2}{*}{1,03004} & 0,4669 \\
\hline $2^{\mathrm{a}}$ amostra $(\mathrm{n}=76)$ & 11,2358 & & \\
\hline Amostras da UNICV & Variância & Estatística $\boldsymbol{F}$ & $\boldsymbol{p}$-value \\
\hline $3^{\mathrm{a}}$ amostra $(\mathrm{n}=32)$ & 10,8096 & \multirow{2}{*}{1,13926} & 0,3926 \\
\hline $4^{\mathrm{a}}$ amostra $(\mathrm{n}=32)$ & 9,4883 & & \\
\hline
\end{tabular}

Fonte: Dados da pesquisa.

Na percepção dos alunos da UFRN e da UNICV, os relatórios financeiros padrão CVM e padrão europeu são iguais quanto à forma adequada de apresentação. A Tabela 9 mostra o resultado do teste $\mathrm{F}$ para a hipótese nula de que as variâncias das populações são iguais, com p-valor $\geq 0,2561$ e 0,3147 , respectivamente. 
Tabela 9 - Forma adequada do relatório

\begin{tabular}{c|c|c|c}
\hline Amostras da UFRN & Variância & Estatística $\boldsymbol{F}$ & $\boldsymbol{p}$-value \\
\hline $1^{\mathrm{a}}$ amostra $(\mathrm{n}=76)$ & 15,5078 & \multirow{2}{*}{1,2671} & 0,2561 \\
\hline $2^{\mathrm{a}}$ amostra $(\mathrm{n}=76)$ & 12,2392 & & Estatística $\boldsymbol{F}$ \\
\cline { 1 - 2 } Amostras da UNICV & Variância & \multirow{2}{*}{1,2597} & 0,3147 \\
\hline $3^{\mathrm{a}}$ amostra $(\mathrm{n}=32)$ & 11,3925 & & \\
\hline $4^{\mathrm{a}}$ amostra $(\mathrm{n}=32)$ & 9,04386 & &
\end{tabular}

Fonte: Dados da pesquisa.

Em relação ao fato de o usuário preferir outro tipo de apresentação do relatório, a tabela 10 mostra o resultado da ANOVA para a hipótese nula de que as variâncias das populações são iguais ao $p$-valor $\geq 0,0011$. O resultado sugere que na percepção dos alunos brasileiros os relatórios financeiros padrão CVM e padrão europeu são diferentes quanto a preferir outro tipo de relatório, evidenciando o indício do efeito propriedade. $\mathrm{O}$ mesmo também se observa para a amostra com os alunos cabo-verdianos, $\mathrm{p}$-valor $\geq 0,0299$.

Tabela 10 - O usuário prefere outro tipo de apresentação do relatório

\begin{tabular}{c|c|c|c}
\hline Amostras da UFRN & Variância & Estatística $\boldsymbol{F}$ & $\boldsymbol{p}$-value \\
\hline $1^{\mathrm{a}}$ amostra $(\mathrm{n}=76)$ & 19,5437 & \multirow{2}{*}{3,0996} & 0,0011 \\
\hline $2^{\mathrm{a}}$ amostra $(\mathrm{n}=76)$ & 6,3053 & & \\
\cline { 1 - 2 } Amostra da UNIVC & Variância & Estatística $\boldsymbol{F}$ & $\boldsymbol{p}$-value \\
\hline $3^{\mathrm{a}}$ amostra $(\mathrm{n}=32)$ & 3,7422 & \multirow{2}{*}{2,4960} & 0,0299 \\
\hline $4^{\mathrm{a}}$ amostra $(\mathrm{n}=32)$ & 9,3406 & & \\
\hline
\end{tabular}

Fonte: Dados da pesquisa.

\section{Considerações Finais}

A pesquisa busca responder a diversos questionamentos sobre o comportamento do usuário da informação contábil, relacionados a questões referentes à teoria da subordinação à forma. Especificamente, se a avaliação da forma como são apresentadas as demonstrações contábeis influenciam os usuários externos da contabilidade.

Em relação ao efeito propriedade, buscou-se identificar se os usuários das informações contábeis tendem a preferir escolher o modelo de representação das demonstrações que já conhecem. Tal evento ficou latente pelos resultados apresentados na tabela 10, em função das preferências dos alunos pelos relatórios pátrios, em que há aceitação das demonstrações da CVM por parte dos estudantes brasileiros e os cabo-verdianos são afeitos aos de seu país, corroborando os achados de Cardoso e Riccio (2005), Cardoso et al. (2007) e Silva e Lima (2007).

Da análise da pesquisa, pode-se inferir que para as amostras brasileiras e caboverdianas, os resultados sugerem a presença do efeito propriedade em ambas as amostras, confirmando que o usuário não prefere outro tipo de representação gráfica do relatório financeiro, mas sim aquele que ele já conhece e domina, confirmando o previsto no Quadro 1, com p-valor $\geq 0,0011$ e 0,0004 , respectivamente.

Em referência à subordinação à forma, não foram encontradas evidências significantes estatisticamente para aceitar a hipótese de que a forma como as demonstrações são apresentadas influencia no julgamento dos usuários das informações para ambas as amostras, apresentando evidências que o efeito framing não se denota determinante para essas tomadas 
de decisões. Entretanto, foram encontradas evidências significantes estatisticamente de que a forma pode influenciar na compreensão do conteúdo informacional apenas para a amostra dos alunos da UNICV com p-valor $\geq 0,0034$, sugerindo a necessidade de novas pesquisas que incorporem questões culturais.

Quanto à análise das demonstrações contábeis, por meio de índices financeiros utilizando-se as duas demonstrações, esperava-se que não houvesse diferença da média nas respostas, mesmo que independente de cálculo (não houve necessidade de cálculo), mas verificou-se que para as amostras com os alunos da UFRN em relação ao Retorno Sobre o Ativo e o Retorno Sobre o Patrimônio Líquido, os resultados sugerem diferenças estatísticas significantes, sinalizando que a forma de apresentação das demonstrações pode afetar a interpretação do usuário. Enquanto que para as amostras com alunos da UNICV não apresentou diferenças estatísticas significantes, conforme o sinal esperado sugerido no Quadro 1 , uma vez que foi esse o comportamento dos alunos da UFRN, revelando o efeito framing dependence.

A pesquisa evidenciou achados quanto à clareza e à objetividade das demonstrações contábeis, sugerindo que existe a necessidade de novos estudos, ampliando-se o tamanho da amostra e também de aplicação deste modelo de pesquisa em outros países.

Como sugestão para trabalhos futuros, indicamos a replicação da pesquisa em países europeus, bem como a aplicação de uma pesquisa com o objetivo de verificar se a forma como as demonstrações contábeis são apresentadas afetam o comportamento dos usuários quanto ao resultado dos índices financeiros na análise fundamentalista.

\section{Referências}

ARIELY, D. Previsivelmente Irracional. Como as situações do dia-a-dia influenciam as nossas decisões. São Paulo: Campus, 2008.

BAKER, H. K.; NOFSINGER, J. R. Psychological Biases of Investors. Financial Services Review. v. 11, n. 2, p. 97-116, 2002.

BARBER, B. M.; ODEAN, T. The Courage of misguided convictions. Financial Analysts Journal. v. 55, n. 6, p. 41-55, 1999.

BAZERMAN, Max H. Processo Decisório: para cursos de administração e economia. Tradução de Arlete Símile Marques. Rio de Janeiro: Elsevier, 2004.

BELKAOUI, Ahmed. Behavioral Accounting. Westport Connecticut: Quorum Books, 1989.

CARDOSO, R. L.; OYADORMARI, J. C. T.; MENDONÇA NETO, O. R. O Framing effect em ambiente contábil: uma explicação fundamentada na teoria dos modelos mentais probabilísticos. In: Encontro da ANPAD, 31, 2007, Rio de Janeiro. Anais ENANPAD, 2007 CD-ROM. 
CARDOSO, R. L.; RICCIO, E. L. Framing effect em um ambiente de informação contábil: um estudo usando a prospect ttheory. In: Encontro da ANPAD, 29, 2005, Brasília. Anais ENANPAD, 2005 CD-ROM.

COOPER, Donald R.; SCHINDLER, Pamela S. Métodos de Pesquisa em Administração. 7. ed. Porto Alegre: Bookman, 2003.

DEHAENE, Stanislas. Varieties of numerical abilities. Cognition, v. 44, n. 1-2, p. 1-42, 1992.

DEHAENE, Stanislas; DUPOUX, Emmanuel; MEHLER, Jacques. Is numerical comparison digital? Analog and symbolic effects in two-digit number comparison. Journal of Experimental Psychology. Human Perception and Performance, v. 16, n. 3, p. 626-641, 1990.

GERLETTI, S.; SAUAIA, A. C. A. Influências de aspectos cognitivos sobre decisões de marketing: Um estudo exploratório sobre decisões de preço em um ambiente negocial simulado. XI SEMEAD, 2008.

GIL, A. C. Como elaborar projetos de pesquisa. 4. ed. São Paulo: Atlas, 2002. HENDRIKSEN, Eldon S.; VAN BREDA, Michael F. Teoria da Contabilidade. São Paulo: Atlas, 1999.

HINRICHS, James V.; YURKO, Dales S.; HU, Jing-Mei. Two-digit number comparison: use of place information. Journal of Experimental Psychology. Human Perception and Performance, v. 7, n. 4, p. 890-901, 1981.

KAHNEMAN, Daniel; TVERSKY, Amos. Prospect theory: an analysis of decision under risk. Econometrica, v. 47, n. 2, p. 263-291, 1979.

KIMURA, Herbert. Aspectos Comportamentais Associados às Reações do Mercado de Capitais. RAE-Eletrônica. v. 2, n. 1, s./p. jan./jun. 2003.

LIBBY, Robert; BLOOMFIELD, Robert; NELSON, Mark W. Experimental research in Finalcial Accounting. Accounting, Organizations and Society. v. 27, p. 775-810, 2002.

MATIAS-PEREIRA, José. Manual de metodologia da pesquisa científica. 2. Ed. São Paulo: Atlas, 2010.

MCCLOSKEY, Michael. Cognitive mechanisms in numerical processing: evidence from acquired dyscalculia. Cognition. v. 44, p. 107-157, 1992.

MCCLOSKEY, Michael; CARAMAZZA, Alfonso; BASILI, Annamaria. Cognitive mechanisms in number processing and calculation: evidence from dyscalculia. Brain and Cognition. v. 4, p. 171-196, 1985.

MCCLOSKEY, Michael; MACARUSO, Paul. Representing and using numerical information. American Psychologist. v. 50, n. 5, p. 351-363, may. 1995. 
NASCIMENTO, A. R.; RIBEIRO, D. C.; JUNQUEIRA, E. R. Estado da arte da abordagem comportamental da contabilidade gerencial: Análise das pesquisas internacionais. In: $8^{\circ}$ congresso USP de controladoria e contabilidade. São Paulo, 2008.

RUTLEDGE, R. W. The ability to moderate recency effects through framing of management accounting information. Journal of management Issues. v. 7, n. 1, Spring, 1995, p. 27-40.

SIEGEL, Gary; RAMANAUSKAS-MARCONI, Helene. Behavioral Accounting. Cincinnati, Ohio: South-Western Publishing Co., 1989.

SILVA, C. A. T.; LIMA, D. H. S. Formulation effect: influência da forma de apresentação sobre o processo decisório de usuários de informações financeiras. In: Encontro da ANPAD, 31, 2007, Rio de janeiro. Anais ENANPAD, 2007 CD-ROM.

SILVA, Edna Lucia da; MENEZES, Estera Muszkat. Metodologia da pesquisa e elaboração de dissertação. 4. ed. Florianópolis: UFSC, 2005. 138 p. Disponível em:

$<$ http://www.portaldeconhecimentos.org.br/index.php/por/content/view/full/10232>. Acesso em: 23 dez. 2012.

SIMON, Herbert A. Comportamento Administrativo: Estudo dos Processos Decisórios nas Organizações Administrativas. Rio de Janeiro: Centro de Publicações Técnicas da Aliança para o Progresso, 1965.

SIMON, Herbert A. Rational decision making in business organizations. American Economic Review. v. 69, n. 4, p. 493-513, Sep. 1979.

SPRINGER, C. W.; BORTHICK, A. F. Improving performance in accounting: evidence for insisting on cognitive conflict tasks. Issues in accounting Education. v. 22, n. 1, p. 1-19, Feb. 2007.

TVERSKY, Amos; KAHNEMAN, Daniel. The framing of decisions and the psychology of choice. Science, v. 211, p. 453-458. Jan. 1981.

VANHUELE, Marc; DRĖZE, Xavier. Measuring the price knowledge shoppers bring to the store. Journal of Marketing. v. 66, p. 72-85, oct. 2002. 